\title{
Pandemics, Public Health Interventions and Fertility: Evidence from the 1918 Influenza
}

\author{
Sander Wagner ${ }^{\mathrm{a}}$, Felix C. Tropf ${ }^{\mathrm{a}, \mathrm{b}}$, Nicolò Cavalli ${ }^{\mathrm{b}, \mathrm{c}, \mathrm{d}}$, and Melinda C. Mills $\mathrm{s}^{\mathrm{b}, \mathrm{c}}$ \\ ${ }^{a}$ École Nationale de la Statistique et de l'Administration Économique, Center for \\ Research in Economics and Statistics, Palaiseu, 91764 France \\ ${ }^{\mathrm{b}}$ Nuffield College, University of Oxford, Oxford, OX1 1NF United Kingdom \\ ${ }^{\mathrm{c}}$ Leverhulme Centre for Demographic Science, University of Oxford, Oxford, OX1 \\ 3UQ United Kingdom \\ ${ }^{\mathrm{d}}$ Department of Social and Political Sciences, Università Commerciale Luigi \\ Bocconi, Milano, 20136 Italy
}

\begin{abstract}
Understanding how pandemics - and the policy interventions to counter them - interact with demographic processes is of urgent scientific importance. Leveraging vital records from 26 major cities in the United States, we assess how variation in non-pharmaceutical interventions (NPIs), during the 1918 influenza pandemic influenced fertility. We find a strong reduction in fertility 9 months after pandemic peaks, with fertility dropping $20 \%$ in cities with short NPIs. Fertility a year later returns to pre-pandemic levels only in the cities with longer implementation of NPIs. The interplay between longer NPIs and reduced virus transmission appears to be the main mechanism increasing fertility 9 months after implementation.
\end{abstract}

\section{Introduction}

Non-pharmaceutical public health interventions (NPIs), such as school closures, mandated physical distancing, and shelter-in-place are important public policy tools to reduce viral transmission during pandemic outbreaks. Epidemiological evidence shows that NPIs were successful at reducing mortality rates during the $1918 \mathrm{H} 1 \mathrm{~N} 1$ influenza A, known as the 'Spanish flu' pandemic in the United States $[1,2]$ and led to eductions in the spread of severe acute respiratory syndrome-coronavirus 2 (SARSCoV-2) during the ongoing COVID-19 pandemic [3].

The demographic consequences of COVID-19 have largely focussed on aspects such as age-specific mortality [4], with limited understanding of fertility consequences. Yet there is considerable debate as to whether shelter-in-place orders and other NPIs will lead to a baby boom or bust. Findings on the effect of pandemics and natural disasters on fertility have been mixed. Although a baby boom 
from the 1965 blackout was widely heralded in the media, later research found no marked increase in births [5]. Severe hurricanes were shown to negatively impact fertility, yet for every 24 additional hours individuals fell under less severe hurricane warnings, a small increase in births was found [6].

Given that data to study fertility outcomes is not yet available for the current pandemic, we can learn from and leverage historical data. A drop in births was found in France following the 1889 influenza [7] and after the 1918 Spanish flu in Sweden [8]. A study of fertility in the United States following the 1918 Spanish flu discovered differences in relation to peak mortality, with a drop in births 3 months after the peak, reversion to normal levels 5-7 months after and a drop 9-10 months after peak mortality [9]. Yet this study only examined variation at the state level and did not interrogate differences in NPIs across cities. Experts anticipate that COVID-19 will result in a baby bust followed by a stronger period of recovery [10], but little attention has been paid to the impact of public health interventions on fertility.

The current study leverages historical data with NPIs of differing lengths across major cities in the US during the 1918-19 Spanish influenza. We use data and definitions based on Markel et al [2], which define NPIs as days in which at least one of the three major interventions: school closures; public gathering bans; and, isolation and quarantine was in place. Understanding the effects of variation in NPIs is highly relevant for COVID-19 since a similar diversity of public health interventions across cities and regions is currently taking place. Previous research demonstrated how variation in NPIs across US cities resulted in variation of transmission and mortality [1] yet we do not know if they impacted on fertility. Understanding and forecasting the longer-term demographic consequences of pandemics and their interplay with NPIs is vital for a variety of policies related to planning child-care, education, housing and the labour market.

\section{Results}

Panel A) of Figure 1 immediately reveals the sharp mortality peak of autumn 1918. In line with previous literature [2] we observe that NPIs effectively 'flattened the curve'. Cities with a longer duration of NPIs recorded significantly reduced peak death rates.

Panel B) shows that mortality peaks were accompanied by an instantaneous decrease in births. This is related to the fact that the 1918 pandemic strongly affected individuals in reproductive ages, leading to higher maternal deaths, miscarriages and stillbirths [9]. Our data further shows fertility troughs around 9 months after the largest and second largest mortality peak in October 1918 and February 1920 respectively.

Panel C) illustrates the relative drop in the Crude Birth Rate ( $\Delta$ CBR) relative to the 1917 average for cities with longer or short NPIs (see Material and Methods). The relative CBR drops from the 1917 average immediately after the pandemic mortality peak in 1918. Both the immediate fertility reduction as well as the fertility drop 9 months after pandemic peaks are more pronounced in cities with shorter NPIs, with fertility dropping by about $20 \%$, compared to $10-12 \%$ in cities with longer NPIs. Cities with more aggressive interventions not only suffer less from a short-term reduction in fertility during the pandemic but also display a more robust rebound to previous fertility levels. Since the negative effect of spiking mortality during a pandemic on subsequent fertility is 
well established, the short-term effects of a NPI could result from either the reduction in mortality that accompanies longer-lasting NPIs or from the direct effects of these interventions on fertility behaviour.

To identify these different channels we applied longitudinal panel analyses with city-level monthly time series of CBR, CDR and whether NPIs were implemented in given months (see Material and Methods).

Results in Table 1 show first that both increased CDR (-0.07 SE 0.01) and the application of NPIs in a given month (-0.07 SE 0.03) have independent significant negative effects on fertility (Model 1). These additional analyses imply that the short-term buffered fertility reductions found in cities with longer NPIs are driven by the reduced mortality levels that accompany NPIs. Model 2 furthermore displays a significant interaction between mortality and NPIs (0.05 SE 0.02), suggesting that during interventions the negative effect of mortality is reduced and in high mortality cities, the negative effect of NPIs diminishes and eventually becomes positive.

\section{Discussion}

We find that the two pandemic peaks of 1918 and 1919 were both followed by an immediate and then second fertility decline 9 months after the peak. Importantly, cities that implemented longer NPIs had lower fertility due to weaker pandemic outbreaks. We can conclude that longer NPIs reduce viral transmission during a pandemic which in turn result in lower mortality peaks and hence less severe impacts on reducing fertility. Several competing mechanisms may operate to explain the relationship between the length of NPIs and fertility. First, NPIs and mortality are signals of the strength of the pandemic. Fear of infection of oneself or family in addition to concerns about lack of healthcare capacity might lead individuals to abstain from childbearing. A second impact of NPIs on fertility may occur via changes in partnership formation, dissolution and behaviour within relationships. Although lockdowns result in more time spent together for cohabiting couples, research has found a reduction in sex during COVID-19 [11], increase in domestic violence and inability to divorce [12]. The limitations to social mixing brought about by stringent NPIs likewise decrease the opportunities to enter into partnerships during the pandemic. A third way in which fertility may be affected is related to lack of access for contraception or assisted reproductive technology (ART) [10], with relevancy for the current pandemic. A fourth inhibitor of fertility are the increased childcare and domestic duties taken on by parents during shelter-in-place measures when for instance, children are not at school or outside of the household. The final and perhaps strongest factor impacting fertility and life planning during a pandemic is the economic recession, unemployment and uncertainty. Cross-national research examining economic recessions and periods of uncertainty has demonstrated that economic and employment uncertainty are key in postponed and lower fertility [13]. Our findings demonstrate that pandemics do not result in a baby boom, but rather a baby blip. More stringent NPIs operate to buffer a potentially strong post-pandemic baby bust. Although we can learn lessons from the Spanish flu, historical comparisons should be approached with caution, since over the past century, societies have undergone major changes [10]. Differences include the widespread use of contraception, access to ART, online dating [14] and adoption of digital communication technologies 
which have changed family patterns [15]. It is possible that, today, negative fertility effects of lockdown are buffered by maintaining some degrees of digital social mixing and higher levels of employment. Fertility replacement due to high levels of child mortality is also negligible in the COVID-19 age. Nevertheless, our study helps to characterise the potential demographic impacts of the implementation of NPIs .

\section{Material and Methods}

To compute crude death and birth rates (CDR and CBR per 1000), we digitized the monthly number of deaths and births from 1917-1922 available from the National Center for Health Statistics at the Center for Disease Control and Prevention as well as information on the total population size per city available for 1917 and 1920-22, estimated on July 1st in each respective year.

We extrapolated monthly population size based on birth and death figures upwards from 1917 on and calculated expected monthly migration by comparing the deviation of extrapolated and measured values.

We combined these vital statistics with secondary source information on NPI intensity, defined as the summed duration in days of different NPI measures, implemented in each of these 26 cities. This dataset was originally compiled based on municipal health department bulletins, local newspapers, and pandemic reports [2].

The cities experienced NPIs between 39 and 164 days (median 60 days) during the second wave of the pandemic, starting September 1918. They are Albany, NY (47 NPI days), Baltimore, MD (43), Boston, MA (50), Buffalo, NY (49), Cambridge, MA (49), Cleveland, OH (99), Columbus, OH (147), Dayton, OH (156), Fall River, MA (60), Grand Rapids, MI (62), Indianapolis, IN (82), Louisville, KY (145), Lowell, MA (59), Milwaukee, WI (132), Minneapolis, MN (116), New Haven, CT (39), New York City, NY (73), Philadelphia, PA (51), Pittsburgh, PA (53), Richmond, VA (60), Rochester, NY (54), Spokane, WA (164), Syracuse, NY (39), Toledo, OH (102), Washington, DC (64), Worcester, MA (44).

We calculated trends of CBR and CDR as well as deviations from the average 1917 fertility. Deviations $\triangle C B R_{M, N P I}=\frac{C B R_{M, N P I}-C \bar{B} R_{1917, N P I}}{C B R_{1917, N P I}}$ tell us by what percentage fertility in high and low NPI cities deviates from their average fertility prior to the pandemic. NPI is a dummy for whether a city falls into the long or short NPI group, $C B R_{M, N P I}$ is the average birth rate of cities within a NPI group in month $M$ and $C \bar{B} R_{1917, N P I}$ is the average birth-rate throughout 1917.

The regression model displayed in Table 1 is specified as:

$C B R_{M, C}=\alpha+\beta_{1} C D R_{M-9, C}+\beta_{2} N P I_{M-9, C}+\beta_{3} C D R_{M-9, C} * N P I_{M-9, C}+\delta M+\gamma_{C}+\epsilon_{M, C}$ where $C B R_{M, C}$ is the Crude Birth Rate in Month $M$ and city $C, C D R_{M-9, C}$ and $N P I_{M-9, C}$ are measures of the crude death rate and a dummy for whether a city was currently implementing an NPI with a 9 month lag, $\delta M$ captures the time trend and $\gamma C$ are city-level fixed effects. The model was estimated both with and without the interaction effect captured by beta $a_{3}$. 
Table 1: Mortality and Lockdown Effects on Fertility 9 Months later

\begin{tabular}{|c|c|c|}
\hline & (1) & (2) \\
\hline CDR & $\begin{array}{c}-0.07^{* * *} \\
(0.01)\end{array}$ & $\begin{array}{c}-0.10^{* * *} \\
(0.02)\end{array}$ \\
\hline NPI & $\begin{array}{c}-0.07^{*} \\
(0.03)\end{array}$ & $\begin{array}{c}-0.16^{* *} \\
(0.05)\end{array}$ \\
\hline $\mathrm{CDR} \times \mathrm{NPI}$ & & $\begin{array}{l}0.05^{*} \\
(0.02)\end{array}$ \\
\hline Constant & $\begin{array}{c}1.16^{* * *} \\
(0.12) \\
\end{array}$ & $\begin{array}{c}1.14^{* * *} \\
(0.12)\end{array}$ \\
\hline Observations & 1638 & 1638 \\
\hline Adjusted $R^{2}$ & 0.06 & 0.06 \\
\hline \multicolumn{3}{|c|}{$\begin{array}{l}\text { Standard errors in parentheses } \\
{ }^{*} p<0.05,{ }^{* *} p<0.01,{ }^{* * *} p<0.001 \\
\text { Dependent Variable is Crude Birth Rate (CBR) } \\
\text { CDR denotes Crude Death Rate measured } 9 \text { months prior to CBR }\end{array}$} \\
\hline
\end{tabular}
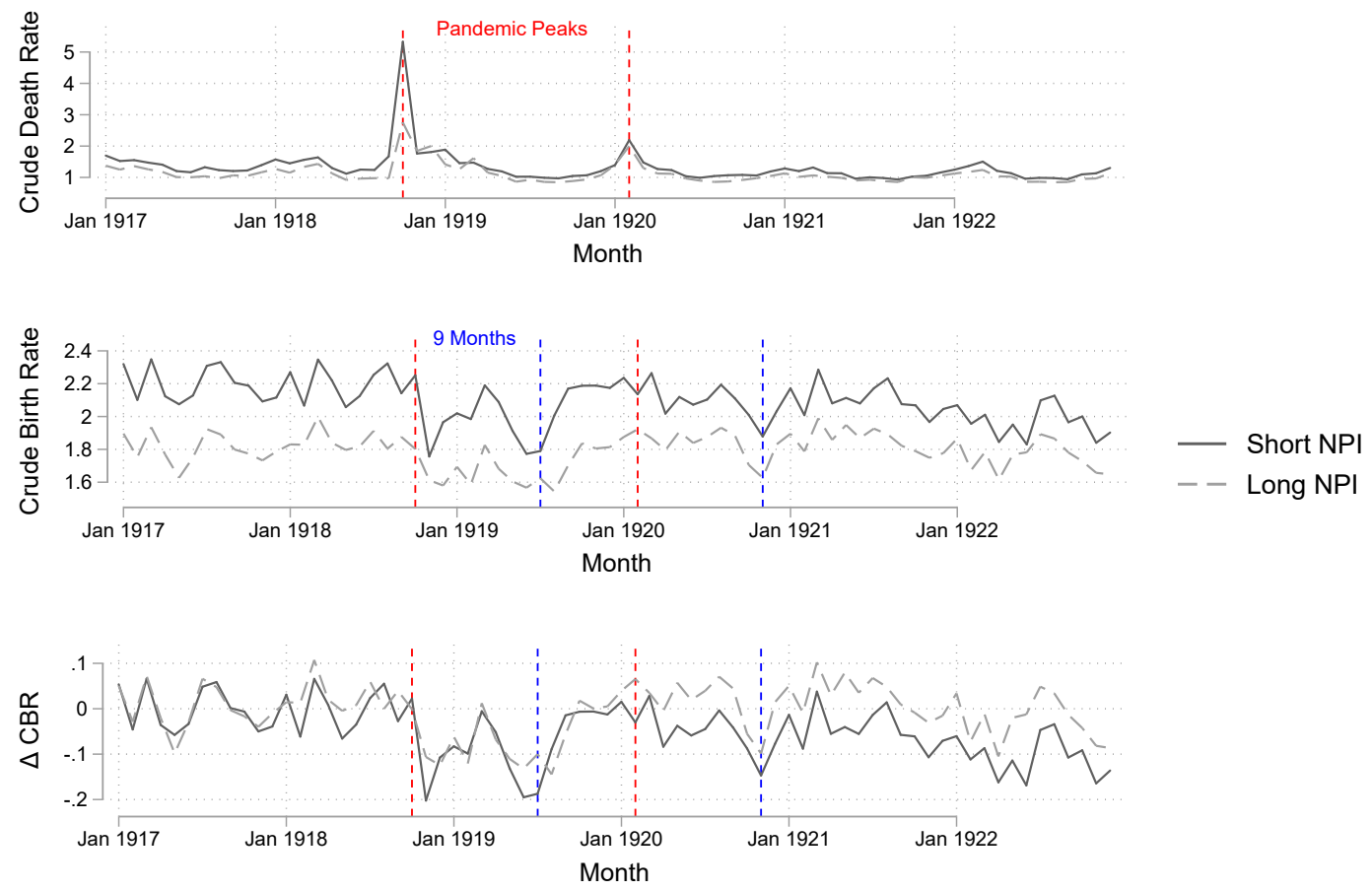

Figure 1: City level demeaned Crude Death (A) and Birth (B) and standardized Birth Rates (C) per 1,000 residents in 26 United States cities, 1917 to 1922. 


\section{References}

[1] M. C. J. Bootsma and N. M. Ferguson, "The effect of public health measures on the 1918 influenza pandemic in u.s. cities," Proceedings of the National Academy of Sciences, vol. 104, no. 18, pp. 7588-7593, 2007, ISSN: 0027-8424. DOI: 10.1073/pnas.0611071104. eprint: https : //www. pnas.org/content/104/18/7588.full.pdf. [Online]. Available: https://www . pnas. org/content/104/18/7588.

[2] H. Markel, H. B. Lipman, J. A. Navarro, A. Sloan, J. R. Michalsen, A. M. Stern, and M. S. Cetron, "Nonpharmaceutical Interventions Implemented by US Cities During the 1918-1919 Influenza Pandemic," JAMA, vol. 298, no. 6, pp. 644-654, Aug. 2007, ISSN: 0098-7484. DOI: 10. 1001/jama . 298.6.644. eprint: https : / jamanetwork. com/journals/jama/articlepdf / 208354/joc70085\_644\_654.pdf. [Online]. Available: https://doi.org/10.1001/jama. 298.6 .644$.

[3] S. Flaxman, S. Mishra, A. Gandy, H. Unwin, T. Mellan, H. Coupland, C. Whittaker, H. Zhu, T. Berah, J. Eaton, M. Monod, A. Ghani, C. Donnelly, S. Riley, M. Vollmer, N. Ferguson, L. Okell, S. Bhatt, P. Perez-Guzman, and P. Walker, "Estimating the effects of non-pharmaceutical interventions on covid-19 in europe," Nature, vol. 584, Aug. 2020. DOI: 10.1038/s41586-0202405-7.

[4] J. B. Dowd, L. Andriano, D. M. Brazel, V. Rotondi, P. Block, X. Ding, Y. Liu, and M. C. Mills, "Demographic science aids in understanding the spread and fatality rates of covid-19," Proceedings of the National Academy of Sciences, vol. 117, no. 18, pp. 9696-9698, 2020, ISSN: 0027-8424. DOI: 10.1073/pnas . 2004911117. eprint: https ://www . pnas .org/content/117/ 18/9696.full.pdf. [Online]. Available: https://www.pnas.org/content/117/18/9696.

[5] J. R. Udry, "The effect of the great blackout of 1965 on births in new york city," Demography, vol. 7, no. 3, pp. 325-327, 1970, ISSN: 00703370, 15337790. [Online]. Available: http://www. jstor.org/stable/2060151.

[6] R. Evans, Y. Hu, and Z. Zhao, "The fertility effect of catastrophe: U.s. hurricane births," Journal of Population Economics, vol. 23, Sep. 2007. DOI: 10.1007/s00148-008-0219-2.

[7] J. Berillon, Annuaire statistique de la ville de Paris pour l'année 1890. 1892.

[8] N. Boberg-Fazlic, M. Ivets, M. Karlsson, and T. Nilsson, "Disease and Fertility: Evidence from the 1918 Influenza Pandemic in Sweden," Research Institute of Industrial Economics, Working Paper Series 1179, Aug. 2017. [Online]. Available: https ://ideas.repec.org/p/ hhs/iuiwop/1179.html.

[9] S. Chandra, J. Christensen, S.-E. Mamelund, and N. Paneth, "Short-Term Birth Sequelae of the 1918-1920 Influenza Pandemic in the United States: State-Level Analysis," American Journal of Epidemiology, vol. 187, no. 12, pp. 2585-2595, Jul. 2018, ISSN: 0002-9262. DOI: 10. 1093/aje/kwy153. eprint: https : / /academic . oup.com/aje/article-pdf/187/12/ 2585/26927868/kwy153.pdf. [Online]. Available: https://doi.org/10.1093/aje/kwy153. 
[10] A. Aassve, N. Cavalli, L. Mencarini, S. Plach, and M. Livi Bacci, "The covid-19 pandemic and human fertility," Science, vol. 369, no. 6502, pp. 370-371, 2020, ISSN: 0036-8075. DOI: 10. 1126/science . abc9520. eprint: https : / / science . sciencemag . org/content/369/ 6502/370.full.pdf. [Online]. Available: https://science.sciencemag.org/content/369/ $6502 / 370$.

[11] J. J. Lehmiller, J. R. Garcia, A. N. Gesselman, and K. P. Mark, "Less sex, but more sexual diversity: Changes in sexual behavior during the covid-19 coronavirus pandemic," Leisure Sciences, vol. 0, no. 0, pp. 1-10, 2020. DOI: 10.1080/01490400.2020.1774016. eprint: https: //doi.org/10.1080/01490400.2020.1774016. [Online]. Available: https://doi.org/10 . $1080 / 01490400.2020 .1774016$.

[12] E. Leslie and R. Wilson, "Sheltering in place and domestic violence: Evidence from calls for service during covid-19," Journal of Public Economics, vol. 189, p. 104241 , 2020, ISSN: 00472727. DOI: https : / / doi . org/10 . 1016/j . jpubeco . 2020 . 104241. [Online]. Available: http://www.sciencedirect.com/science/article/pii/S0047272720301055.

[13] N. Balbo, F. C. Billari, and M. Mills, "Fertility in advanced societies: A review of research," European Journal of Population, vol. 29, no. 1, pp. 1-38, 2013, ISSN: 01686577, 15729885. [Online]. Available: http://www.jstor.org/stable/42636100.

[14] M. J. Rosenfeld, R. J. Thomas, and S. Hausen, "Disintermediating your friends: How online dating in the united states displaces other ways of meeting," Proceedings of the National Academy of Sciences, vol. 116, no. 36, pp. 17753-17 758, 2019.

[15] F. C. Billari, O. Giuntella, and L. Stella, "Does broadband internet affect fertility?" Population studies, vol. 73, no. 3, pp. 297-316, 2019. 